\title{
Every moment counts: smooth transitions of object boundaries reflect constant updating in object-based attention
}

\author{
San-Yuan Lin $\cdot$ Su-Ling Yeh
}

Published online: 14 December 2011

(C) Psychonomic Society, Inc. 2011

\begin{abstract}
Detection of a target is faster when it is presented on an attended rather than an unattended object (i.e., objectbased attention). Using the double-rectangle cuing paradigm (Egly, Driver, \& Rafal, 1994), we previously showed that object-based attention operates on a dynamic representation: Object-based attentional guidance is affected by subsequent changes occurring to an attended object (Lin \& Yeh, 2011). The present study provides further support for our dynamicupdating hypothesis, demonstrating that changes are indeed updated into the existing object representation. After a cue display, we introduced changes between the initial display (four hashes) and the final display (four squares with an occluder between them). We found object-based attention only with smooth transitions between the initial and final displays, not when transitions were in the reverse order (Exp. 1) or contained any single disruption of the smooth transition (Exp. 2). These results confirm our hypothesis that attention operates on a dynamic object representation that is constantly and continuously updated.
\end{abstract}

Keywords Object-based attention · Spatial cuing · Object representation $\cdot$ Boundary change

We face a vast amount of information flow in our visual world with only limited capacity, so attention is necessary to select relevant information for further processing. Past studies have shown that in addition to spatial location (Duncan,

S.-Y. Lin $\cdot$ S.-L. Yeh $(\bowtie)$

Department of Psychology, Graduate Institute of Brain and Mind

Science, Neurobiology and Cognitive Science Center,

National Taiwan University,

No. 1, Sec. 4, Roosevelt Road,

Taipei 10617, Taiwan

e-mail: suling@ntu.edu.tw
1984; Vecera \& Farah, 1994), visual attention can also select information on the basis of perceptual objects (Eriksen \& Yeh, 1985; Posner, Snyder, \& Davidson, 1980). Egly, Driver, and Rafal, (1994) used a now-popular doublerectangle paradigm to demonstrate object-based attention. When one's attention is directed to part of an object by a spatial cue at one end of a rectangle, target detection is faster on the cued than on the uncued rectangle when the cue-totarget distance is equal in the two conditions.

While the mechanisms that give rise to such object-based attention are still under debate, recent studies (e.g., Chen \& Cave, 2008; Ho \& Yeh, 2009; Lamy \& Egeth, 2002; Richard, Lee, \& Vecera, 2008; Shomstein \& Behrmann, 2008; Shomstein \& Yantis, 2002) have also suggested that - besides attention itself - the strength of object representation can also influence the deployment of attention on the existing object representation. For example, Shomstein and Behrmann showed that object-based attention was affected by the preview time of the initial object display: When the preview time was long, both the object configuration and the probability of target occurrence affected object-based attention. When the preview time was short, however, the effect of object configuration disappeared because the strength of the object representation was not sufficient to guide attention.

To further explore whether or not changes in an object's representation affect the allocation of attention, we (Lin \& Yeh, 2011) adapted Egly et al.'s (1994) double-rectangle paradigm and showed that object-based attention can be influenced by an attended and later changed object (i.e., four separated squares were changed into two oblong rectangles via amodal completion). In that study, we presented four small squares in the initial display instead of two rectangles. The four squares were placed equidistant to each other, forming the corners of a larger (imaginary) squaresimilar to the display in Frame 4 of the smooth-transition 
condition in Fig. 1. After one of the squares was brightened as a cue, a target was presented. Critically, along with the presentation of the target, an occluder was presented concurrently to induce amodal completion: The four-square configuration was then perceived as a double-rectangle configuration, similar to that in Egly et al.'s study, but with an occluder on top of the two rectangles (see the target-andoccluder display in Fig. 1). Had object-based attention operated in a feed-forward way, accruing its processing from lower to higher levels in the visual system, changes made after the cue presentation should not have a chance to affect the already ongoing attentional processing triggered by the cue. Following this reasoning, the results of Lin and Yeh showed that object-based attention was indeed influenced by the object configuration that was changed at the moment of target presentation, rather than relying entirely on the originally cued object configuration. This indicates that attentional selection can be based on a dynamic object representation that can be changed even after attention has been cued to a particular object representation.

Note that the object change manipulation in Lin and Yeh (2011) was limited to the time frame around the target presentation; thus, it remains unknown whether such a change can be updated into the existing object representation throughout a whole trial. Because Lin and Yeh changed the representation by adding an occluder in the last frame, the change either could be integrated into the existing object representation, as we suggested (i.e., updating), or could overwrite the existing object representation to rebuild it (i.e., overwriting). Both operations could lead to the observed result - that is, the later, changed object determines object-based attention - but each operation suggests that a different process would support and maintain the object representation. To differentiate these two possibilities, we reasoned that if an overwriting process rebuilds the object representation each time a change occurs, then it would not be important how a cued object was changed. However, if an updating process integrates changes continuously into the existing representation, then how the cued object was changed would be important. Therefore, if we manipulated how the object was changed (i.e., the history of the cued object) to see how this affected object-based attention, we could differentiate these two possibilities. In the present study, we examined this issue by introducing boundary changes after the cue display to test whether the history of the cued object would affect object-based attention.

\section{Experiment 1}

In this experiment, we designed different object configurations for the initial display (hashes) and the target display (squares) that underwent boundary changes. An occluder was added in the last frame, following closely the displays

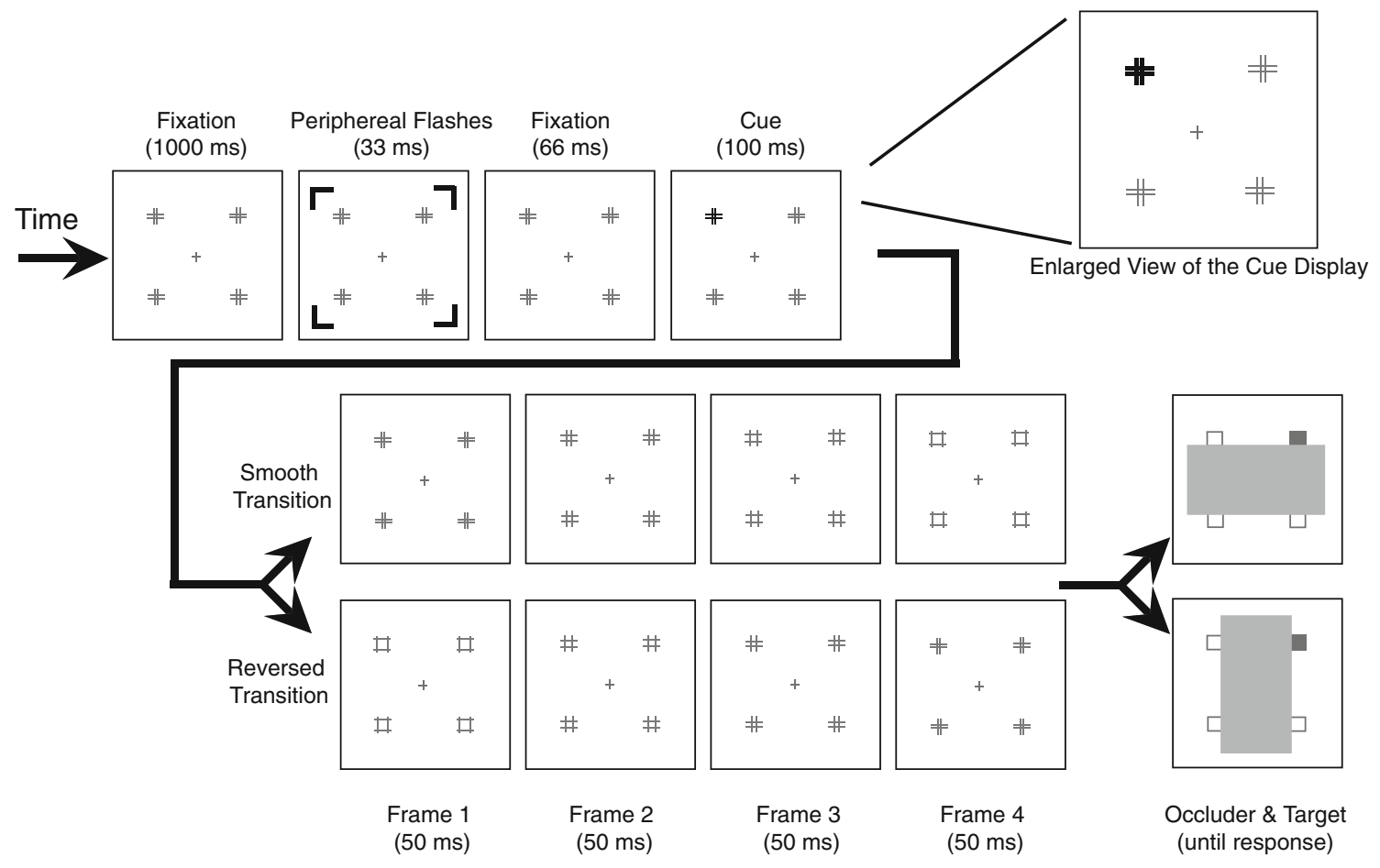

Fig. 1 Stimuli and procedure of Experiment 1. After the cue display, the objects (hashes) underwent transitions to become squares, either smoothly or in a reversed order. The occluder that induced amodal completion appeared simultaneously with the final squares 
used in Lin and Yeh (2011). The critical manipulation lay in the changes between the initial and target displays. Two kinds of transitions were used to link the object configurations: a smooth transition or a reversed-order one. If the representation were overwritten and rebuilt each time a change occurred, no difference should be found in the two transition conditions. In contrast, if the object representation were updated with the constraint of a smooth and continuous change, we should expect to find object-based attention only in the smooth-transition, and not the reversed-order, condition.

\section{Method}

Participants A group of 40 students from National Taiwan University participated in this experiment. All had normal or corrected-to-normal vision and were naïve as to the purpose of the experiment.

Apparatus and stimuli The stimuli were displayed on an Eizo F552-M monitor with $640 \times 480$ resolution that was capable of producing 256-level grayscales. The DMDX program (Forster \& Forster, 2003) was used to present the stimuli and collect the timed responses. The participants sat in a dimly lit chamber at a viewing distance of $57 \mathrm{~cm}$ with their head position maintained by a chinrest.

The initial display (Fig. 1) consisted of four objects (hashes, each subtending $1.4^{\circ} \times 1.4^{\circ}$, thickness $0.2^{\circ}$, light gray) with a central plus pattern $\left(0.25^{\circ} \times 0.25^{\circ}\right.$, thickness $0.04^{\circ}$, light gray) as the fixation point. The end-to-end distances of the outer ends of these rectangles were the same $\left(8.8^{\circ}\right)$. The peripheral flash consisted of four L-shaped patterns $\left(2.4^{\circ} \times\right.$ $2.4^{\circ}$, thickness $0.55^{\circ}$, black) to ensure that the initial attentional focusing of participants was expanded to cover the four small rectangles (Goldsmith \& Yeari, 2003; Ho \& Yeh, 2009). The cue was a brightening of one of the hashes. The smooth transition consisted of displacement of the strokes of a hash at a speed of $0.2^{\circ}$ per frame. An occluder $\left(11^{\circ} \times 8^{\circ}\right.$, dark gray) was shown that covered the inner four edges of the squares to create the percept of two rectangles-not in the sense of disparity-induced stereopsis, but in a two-dimensional pictorial depth percept. The target was a filled square $\left(1.4^{\circ} \times 1.4^{\circ}\right.$, light gray $)$ shown as one of the final squares.

Design The cue could appear on any one of the four hashes with equal probability. Depending on the relative positions between the cue and the target, three types of trials were defined: valid trials (the target appeared at the cued location), invalid-same trials (the target appeared on the rectangle that formed an amodally completed rectangle with the cued object), and invalid-different trials (the target appeared on the rectangle that formed the other amodally completed rectangle). The target never appeared on the object that was diagonal to the cued object.

There were 384 trials in total. The 320 target-present trials included 240 valid trials and 80 invalid trials (40 invalid-same trials and 40 invalid-different trials). To prevent a preparatory response, 64 catch trials included no target. The occluder orientation could be either horizontal or vertical between the squares, to create amodal completion. The trials were completely randomized.

Procedure In each trial, the initial display appeared for $1,000 \mathrm{~ms}$, at which point the peripheral flashes appeared briefly for $33 \mathrm{~ms}$, followed by the cue, which appeared on one of the four outer ends for $100 \mathrm{~ms}$. After the cue display, the hashes were presented in a transitional sequence to become squares (Fig. 1). The transitions consisted of four frames (each lasting $50 \mathrm{~ms}$ ) that occurred right after the cue display. Two kinds of transitions were intermixed: In the smooth transition, the hashes changed into squares by expanding the four strokes outwardly, while in the reversed-order transition, the frames from the smooth transition were displayed in the reverse order. Afterward, an occluder appeared that grouped the four separate objects into two rectangles. At the same time that the occluder appeared, the target appeared at one of three locations - valid, invalid-same, or invalid-different-with respect to the cue location. The participants were required to make a speedy detection of whether the target had appeared and to withhold their responses if there was no target.

\section{Results and discussion}

Error trials (misses and false alarms) and anticipatory responses (reaction times shorter than $150 \mathrm{~ms}$ ) were excluded from further analysis, resulting in the removal of $0.5 \%$ and $0.8 \%$ of trials in the smooth and reversed-order transition conditions, respectively. An overall two-way repeated measures analysis of variance (ANOVA) with Transition (smooth and reversed-order) and Trial Type (valid, invalid-same, and invalid-different) as factors was performed. The main effects of transition and trial type were significant $[F(1,39)=5.1, M S E=885.1, p<.05$, Cohen's $f=0.226 ; F$ $(2,78)=9.85, M S E=2,476.5, p<.001$, Cohen's $f=0.38$, respectively]; see Fig. 2. The interaction between transition and trial type was also significant $[F(2,78)=4.26, M S E=$ 538.6, $p<.05$, Cohen's $f=0.23$ ]. A post-hoc Tukey's test showed that in the smooth-transition condition, participants responded more quickly to valid and invalid-same trials than to invalid-different trials $(p s<.01)$. In the reversed- 


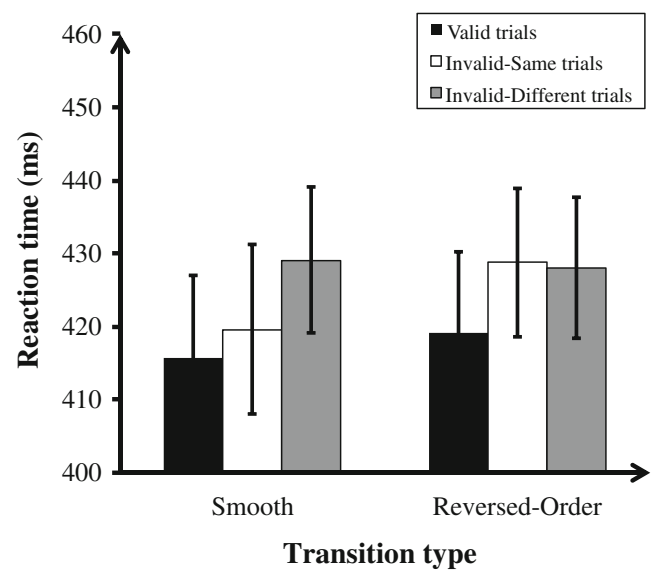

Fig. 2 Experiment 1: As depicted in the graph, the object effect (the difference between the white and gray bars) was significant only when the transition was smooth, suggesting that the history of the object configuration influences object-based attention

order condition, participants responded more quickly to valid than to invalid-same and invalid-different trials $(p \mathrm{~s}<.05)$. These results suggest that the sources of the interaction were faster responding on the valid trials in both conditions and shorter latencies for invalid-same trials in the smooth-transition condition than in the reversed-order condition. That is, while the location effect was found in both conditions, the object effect was only found in the smooth-transition condition, not in the reversed-order condition. In other words, object-based attention can operate only on object representations that are successfully updated via smooth transitions.

The lack of an object effect in the reversed-order transition condition relative to the smooth-transition condition might have resulted from one of the two abrupt changes (Frames 1 and 4 in Fig. 1) or from both. Either of the abrupt changes could have disrupted the object representation, or the disruption could have resulted from the joint action of both of the abrupt changes. We examined these possibilities in the next experiment.

\section{Experiment 2}

The purpose of this experiment was to further investigate the influence of abrupt boundary changes on the updating process. We introduced an abrupt boundary change into either Frame 1 (the initial-abrupt condition) or Frame 4 (the finalabrupt condition) of the smooth-transition condition from Experiment 1, and we used a random transition with shuffled frames from the smooth transition as a baseline (the random condition). If the object effect were obtained in only one of the two abrupt (either initial-abrupt or final-abrupt) conditions, this would suggest different tolerances for abruptness between the early and late stages of the updating process. If the object effect were obtained in both conditions, it would suggest that the updating process has some degree of tolerance to unevenness in the transitions. Finally, if the object effect were absent in both conditions, it would suggest that the updating process requires a smooth change for successful guidance of object-based attention. In any case, we expected to find no object effect in the random condition.

\section{Method}

Participants A second group of 60 naïve participants were tested.

Apparatus, stimuli, design, and procedure The apparatus and stimuli were the same as those in the previous experiment, except that there were three transition conditions: initial-abrupt, final-abrupt, and random. The initial-abrupt condition was prepared by replacing Frame 1 with Frame 4 in the smooth-transition condition of Experiment 1, and the final-abrupt condition by replacing Frame 4 with Frame 1 (Fig. 3). The random transition was prepared by randomizing the four frames on a trial-by-trial basis; the possibility of a smooth transition was excluded from this experiment. There were 576 trials, with the same proportions of trial
Fig. 3 The transitions used in Experiment 2. There were three kinds of transitions: initialabrupt, final-abrupt (as depicted here), and random (not shown) transitions. The random condition was prepared by shuffling randomly the four frames $(1,2$, 3 , and 4) from the smoothtransition condition of Experiment 1

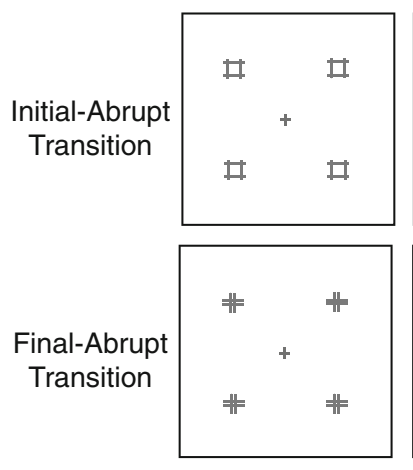

Frame 1

(50 ms)

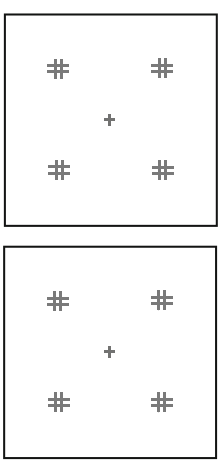

Frame 2

(50 ms)

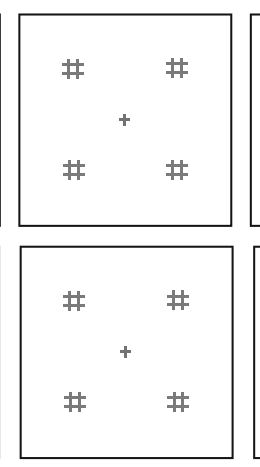

Frame 3

(50 ms)

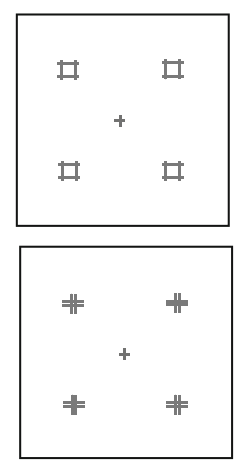

Frame 4

(50 ms) 
types as in Experiment 1-here, however, the invalid-same and invalid-different trials were split evenly among the initial-abrupt, final-abrupt, and random conditions, with 40 trials with each transition per condition.

\section{Results and discussion}

Again, error trials - misses and false alarms - and anticipatory responses (reaction times shorter than $150 \mathrm{~ms}$ ) were excluded from further analysis. This resulted in the removal of $0.42 \%, 0.36 \%$, and $0.36 \%$ of trials in the initial-abrupt, final-abrupt, and random conditions, respectively. An overall two-way repeated measures ANOVA with the factors Transition (initial-abrupt, final-abrupt, and random) and Trial Type (valid, invalid-same, and invalid-different) was performed. The main effect of trial type was significant $[F$ $(2,118)=3.94, M S E=1,231.13, p<.05$, Cohen's $f=0.22]$; see Fig. 4. The main effect of transition and the interaction between transition and trial type were not significant $[F(2$, $118)=1.15, M S E=241.57, p=.32$, and $F(4,236)=0.13$, $M S E=259.79, p=.97$, respectively]. A post-hoc Tukey test showed that participants responded faster to valid trials than to invalid-different trials $(p<.01)$. In the absence of an interaction, these results suggest that there was a location effect but not an object effect when the transitions were either partly disrupted or completely randomized in sequence.

We demonstrated that in both the initial-abrupt and finalabrupt conditions, a single abrupt transition was sufficient to disrupt the object-updating process, and thus object-based attention. This suggests that an updating process was indeed operating on a moment-by-moment basis and that for object-based attention to operate, the object representation should be maintained smoothly through transitions.

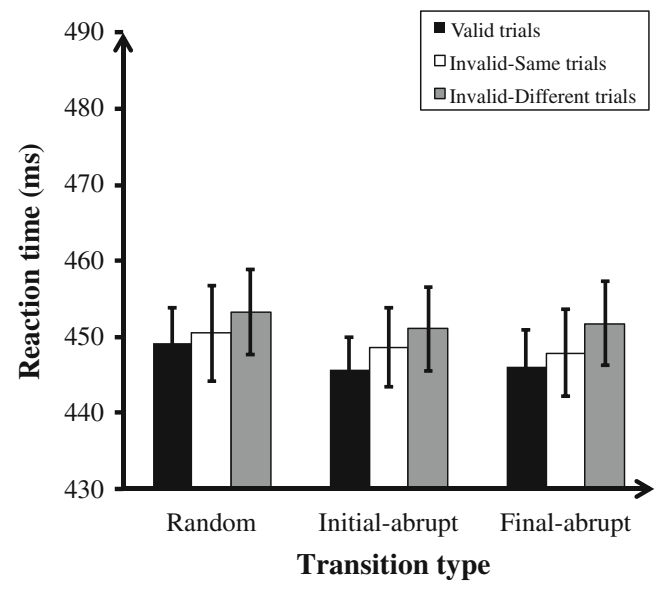

Fig. 4 Experiment 2: There was no object effect when the transition was randomized ("Random") or when an abrupt change was introduced at the initial display of the transition ("Initial-abrupt") or at the end of the transition ("Final-abrupt"), suggesting that a single abrupt change can disrupt the updating process

\section{General discussion}

We previously proposed a dynamic-updating hypothesis to explain the results that an initially attended and then changed object affects object-based attention (Lin \& Yeh, 2011). In the present study, we further demonstrated that the way that the boundary of an object is changed affects objectbased attention. When the intermediate change was either a smooth or a reversed-order transition, object-based attention occurred only when the transition was smooth (Exp. 1). Further investigation of the relative influences of Frames 1 and 4 of the reversed-order condition that had disrupted the object effect in Experiment 1 suggested a continuous updating process that could not tolerate even a single abrupt boundary change (Exp. 2). Altogether, these findings suggest that the object representation under attentional selection is updated - rather than overwritten - each time a change occurs. This result further supports and elaborates the dynamic-updating hypothesis, showing that dynamic updating occurs continuously.

Our findings corroborate the idea that attentional selection on an object is not strictly a feed-forward process, but rather an iterative operation. This could be realized by mechanisms such as a reentrant process (Di Lollo, Enns, \& Rensink, 2000) that operates continuously after the original object is cued. When change occurs - as in Lin and Yeh (2011) and here - the reentrant process is attracted by the change and takes it into effect by integrating the change into the existing representation. That is, when the boundaries of the cued hashes started to expand outward, the change of boundary position induced the reentrant signal to follow the change. When the change was smooth, it was likely to be handed over to neurons representing adjacent receptive fields. Thus, when the higher-level reentrant signal returns to the neurons that signify the change, the signal can integrate this change with the information represented by adjacent neurons. Referring to our displays in the present study, when the hashes finally became squares and then amodally completed to form two larger squares, the subsequent change then occurred at the higher-level stage that involves integration of an object representation and the relations between objects.

In object-based attention, the object and the attention that selects it are closely linked as a dyad. The present study approaches this dyad from an understanding of how an object affects attentional selection. We suggest that there can be at least two levels of object boundary change, if we categorize each change according to whether it involves the object alone or other objects as well. We have demonstrated both types of change in the present study. On the one hand, the change induced by amodal completion at the final target frame was a between-object grouping, because the grouping involved more than one object and the number of objects 
changed after amodal completion (from four to two). On the other hand, the change induced by the linking transitions before target presentation was a within-object change, since it involved only a single object (i.e., the hash itself). That is, two independent changes were present in our displays. While the within-object transition changed the boundaries of individual hashes into squares, the finally presented occluder changed the relation between the hashes (or now squares) from separated objects to parts of larger objects. Had it not been able to tolerate both between-object grouping and the within-object changes after the smooth updating of hashes into squares in the smooth-transition condition, the object effect would not have been observed.

On the basis of the increased size and complexity of receptive fields from lower to higher levels in the visual cortex (Bruce, Desimone, \& Gross, 1981; Hubel \& Wiesel, 1977; Kanwisher, McDermott, \& Chun, 1997) and of the results we obtained here, we suggest that hierarchical object representations have different spatiotemporal properties in response to boundary changes. That is, higher-level representations (i.e., between-object grouping) - as compared to lower-level representations (i.e., within-object change) - are more tolerant of spatiotemporal changes. The small receptive fields at the lower levels can be informative when performing point-to-point correspondence between the boundaries of objects across time. In the present study, the absence of object-based attention for the reversed-order transition (and for all other transitions with abrupt changes) suggests that when the span of boundary changes is larger than the separation of a few receptive fields, the initial and later states cannot be connected by low-level neurons, and thus fail to be represented as changes to the same object. On the other hand, at higher levels of the visual cortex, the location information about features in the object is not as accurate as at lower levels, and thus, the object represented at higher levels is close to a symbolic representation. That is, the between-object grouping changes induced by amodal completion may involve a higher-level merger of the symbolic indexes that are associated with each individual object.

The idea that different levels of object representation can tolerate different extents of spatiotemporal changes further extends object file theory (Kahneman, Treisman, \& Gibbs, 1992): The concept of spatiotemporal continuity should be reconsidered in the light of the hierarchical nature of object representations. The object file theory suggests that the relevant properties of an object are stored together in an intermediate representation that can be retrieved when part of the information of the object is reencountered (Kahneman et al., 1992). Such successful retrieval of the information in the object file is achieved when spatiotemporal continuity is maintained so that different views across time can be treated as views of the same object. As we have demonstrated in this study, we suggest that when the change involves within- object boundary changes, strict spatiotemporal continuity is necessary for successful handling of changes across time. When a change involves between-object grouping, as induced by amodal completion in the last frame, however, the requirement of image-based spatiotemporal continuity can be less stringent: Regrouping between objects, such as that induced by amodal completion, can be abrupt and can occur within a shorter time frame. In other words, we have refined the idea of spatiotemporal continuity by providing boundary conditions that define an object across time at different levels in the hierarchy of object representations. Object continuity is strict in low-level object representations, in the sense that the representation has to be changed continuously in a smooth transition, while transitions can be less smooth at higher levels of representation.

For the other part of the object-attention dyad, the findings of this study also contribute to the theoretical development of how object-based attention operates. Because previous accounts of object-based attention (e.g., the spreading account of Richard et al., 2008; the prioritization account of Shomstein \& Yantis, 2002; and the shift account of Lamy \& Egeth, 2002) were all derived from the use of static objects that were maintained throughout a trial, these theories have difficulties in explaining our finding here that constant changes in the cued object can still be updated into the selected object unit-if there are no further modifications of the theories. For instance, the shifting account suggests that the same-object effect arises from the greater difficulty of shifting attention between objects rather than within a cued object when a target appears (Lamy \& Egeth, 2002). This seems to suggest that attention operates at a higher level of object representation and that the object contour can be less influential: The shifting account would predict that the same-object effect would occur with both the smooth and reversed-order transitions that we manipulated. Thus, to explain our data reported here, accounts based on a static object display would need to incorporate the temporal dimension - that is, how attention operates over time when the object changes.

Finally, our results advance findings that have emphasized the effect of the perceptual strength of object representations on object-based attention (Chen \& Cave, 2008; Lamy \& Egeth, 2002; Richard et al., 2008; Shomstein \& Behrmann, 2008; Shomstein \& Yantis, 2002) by demonstrating the extended influence of perception in the temporal dimension. Our results are in accordance with the work of Shomstein and Behrmann, who showed that prolonging the preview time of the initial display increases the object effect. As we have demonstrated here, as time unfolds in a trial, the object representation can remain to exert its influence not only in the initial display but also until the target presentation. Furthermore, object-based attention can operate not only on a single, uniformly connected region (Watson \& Kramer, 
1999), but also on multiple such regions when they are grouped (Lamy \& Egeth, 2002) — even when the grouping occurs after attention has deployed to the cued object, as shown in the present study. Furthermore, obtaining the object effect only when the transition was smooth and not when the transition contained even a single disruption, we showed the importance of how the object boundaries changed - and thus of the boundaries themselves - in confining object-based attention (Chen \& Cave, 2008; Richard et al., 2008).

In conclusion, this study has demonstrated that boundary changes of an object are constantly updated in the hierarchical object representation. Without the support of successful lower-level updating of smooth changes, a higher-level abrupt change would possibly not be updated so as to influence object-based attention. This characteristic of object representation is important when one tries to understand how an object is represented and how object-based attention operates in a constantly changing world. To successfully achieve behavioral goals, every moment counts.

Author note This research was supported by Taiwan's National Science Council (Grants NSC96-2413-H-002-009-MY3 and NSC 982410-H-002-023-MY3) to S.-L.Y.

\section{References}

Bruce, C., Desimone, R., \& Gross, C. G. (1981). Visual properties of neurons in a polysensory area in the superior temporal sulcus of the macaque. Journal of Neurophysiology, 46, 369-384.

Chen, Z., \& Cave, K. R. (2008). Object-based attention with endogenous cuing and positional certainty. Perception \& Psychophysics, 70, 1435-1443. doi:10.3758/PP.70.8.1435

Di Lollo, V., Enns, J. T., \& Rensink, R. A. (2000). Competition for consciousness among visual events: The psychophysics of reentrant visual processes. Journal of Experimental Psychology: General, 129, 481-507. doi:10.1037/0096-3445.129.4.481

Duncan, J. (1984). Selective attention and the organization of visual information. Journal of Experimental Psychology: General, 113, 501-517. doi:10.1037/0096-3445.113.4.501

Egly, R., Driver, J., \& Rafal, R. D. (1994). Shifting visual attention between objects and locations: Evidence from normal and parietal lesion subjects. Journal of Experimental Psychology: General, 123, 161-177. doi:10.1037/0096-3445.123.2.161
Eriksen, C. W., \& Yeh, Y. (1985). Allocation of attention in the visual field. Journal of Experimental Psychology: Human Perception \& Performance, 11, 583-597. doi:10.1037/0096-1523.11.5.583

Forster, K. I., \& Forster, J. C. (2003). DMDX: A Windows display program with millisecond accuracy. Behavior Research Methods, Instruments, \& Computers, 35, 116-124. doi:10.3758/ BF03195503

Goldsmith, M., \& Yeari, M. (2003). Modulation of object-based attention by spatial focus under endogenous and exogenous orienting. Journal of Experimental Psychology: Human Perception and Performance, 29, 897-918. doi:10.1037/0096-1523.29.5.897

Ho, M.-C., \& Yeh, S.-L. (2009). Effects of instantaneous object input and past experience on object-based attention. Acta Psychologica, 132, 31-39. doi:10.1016/j.actpsy.2009.02.004

Hubel, D. H., \& Wiesel, T. N. (1977). Functional architecture of macaque monkey visual cortex. Proceedings of the Royal Society $B, 198,1-59$.

Kahneman, D., Treisman, A., \& Gibbs, B. J. (1992). The reviewing of object files: Object-specific integration of information. Cognitive Psychology, 24, 175-219. doi:10.1016/0010-0285(92)90007-O

Kanwisher, N., McDermott, J., \& Chun, M. M. (1997). The fusiform face area: A module in human extrastriate cortex specialized for face perception. Journal of Neuroscience, 17, 4302-4311.

Lamy, D., \& Egeth, H. (2002). Object-based selection: The role of attentional shifts. Perception \& Psychophysics, 64, 52-66. doi:10.3758/BF03194557

Lin, S.-Y., \& Yeh, S.-L. (2011). First come, first served? Influence of changed object configuration on object-based attention. Attention, Perception, \& Psychophysics, 73, 678-687. doi:10.3758/s13414010-0078-2

Posner, M. I., Snyder, C. R., \& Davidson, B. J. (1980). Attention and the detection of signals. Journal of Experimental Psychology: General, 109, 160-174. doi:10.1037/0096-3445.109.2.160

Richard, A. M., Lee, H., \& Vecera, S. P. (2008). Attentional spreading in object-based attention. Journal of Experimental Psychology: Human Perception and Performance, 34, 842-853. doi:10.1037/ 0096-1523.34.4.842

Shomstein, S., \& Behrmann, M. (2008). Object-based attention: Strength of object representation and attentional guidance. Perception \& Psychophysics, 70, 132-144. doi:10.3758/PP.70.1.132

Shomstein, S., \& Yantis, S. (2002). Object-based attention: Sensory modulation or priority setting? Perception \& Psychophysics, 64, 41-51. doi:10.3758/BF03194556

Vecera, S. P., \& Farah, M. J. (1994). Does visual attention select objects or locations? Journal of Experimental Psychology: General, 123, 146-160. doi:10.1037/0096-3445.123.2.146

Watson, S. E., \& Kramer, A. F. (1999). Object-based visual selective attention and perceptual organization. Perception \& Psychophysics, 61, 31-49. doi:10.3758/BF03211947 